\title{
Autonomous and Distributed Motion Planning for Satellite Swarm
}

\author{
Dario Izzo* \\ European Space Agency, 2201 AZ Noordwijk, The Netherlands \\ and \\ Lorenzo Pettazzi $\ddagger$ \\ University of Bremen, 28759 Bremen, Germany \\ DOI: $10.2514 / 1.22736$
}

\begin{abstract}
We present a satellite path-planning technique able to make a set of identical spacecraft acquire a given configuration. The technique exploits a behavior-based approach to achieve an autonomous and distributed control over the relative geometry, making use of limited sensorial information. A desired velocity is defined for each satellite as a sum of different contributions coming from generic high-level behaviors. The behaviors are further defined by an inverse-dynamic calculation dubbed equilibrium shaping. We show that by considering only three different kinds of behavior it is possible to acquire a number of interesting formations, and we describe the theoretical framework needed to find the entire set. We find that by allowing a limited amount of communication the technique may be used also to form complex lattice structures. Several control feedbacks able to track the desired velocities are introduced and discussed. Our results suggest that sliding-mode control is particularly appropriate in connection with the developed technique.
\end{abstract}

\section{Introduction}

D OES a coherent group behavior require an explicit mechanism of cooperation? Can useful tasks be accomplished by a homogeneous team of mobile agents without direct communication and using decentralized control? These questions have been addressed by an increasingly large community of computer scientists, engineers and scientists in general working in a field of research that we may call swarm intelligence or collective robotics [1]. The relevance of the possible answers to the aerospace community is significant. Space engineers are currently developing autonomous systems and are envisaging space missions that would certainly benefit from a deeper understanding of the collective behavior of similar and dissimilar agents. Multirobot planetary exploration, on-orbit self-assembly, and satellite swarms for coordinated observations are just examples of what could be achieved if our technology level was proven to be sufficient to provide spacecraft swarms with autonomous decision capabilities. As recently proposed by Ayre et al. [2], it would be possible to build large solar panels or large antennas by exploiting collective emerging behaviours. The use of collective robotics is also very relevant for advanced mission architectures such as those recently studied by ESA (APIES mission [3] ) and NASA (ANTS mission [4]) making use of satellite swarms to explore the asteroid belt.

Because of these synergies between collective robotics and space mission design, it makes sense to try to design a decentralized control system for a satellite swarm, relying upon the lessons learned from collective robotics. Drawing inspiration from this research field we will often use the term "agent" to indicate a particular spacecraft belonging to some group. When a complex system of many agents

Received 25 January 2006; revision received 30 November 2006; accepted for publication 18 October 2006. Copyright (C) 2006 by Dario Izzo and Lorenzo Pettazzi. Published by the American Institute of Aeronautics and Astronautics, Inc., with permission. Copies of this paper may be made for personal or internal use, on condition that the copier pay the $\$ 10.00$ per-copy fee to the Copyright Clearance Center, Inc., 222 Rosewood Drive, Danvers, MA 01923; include the code 0731-5090/07 \$10.00 in correspondence with the CCC

*Research Fellow, Advanced Concepts Team, European Space Research and Technology Centre (ESTEC), Keplerlaan 1; dario.izzo@esa.int.

TPh.D. Candidate, Center of Applied Space Technology and Microgravity (ZARM). has to act in a coordinated manner, the action selected by each agent may or may not take into account the decisions taken by the others. The smaller the number of communications required between the agents, the smaller the degree of coordination of the system. On the other hand, a small amount of communication leads to a simple and robust system. The information exchanged with the other swarm components is useful but not necessary to define the geometric and kinematical representation of the time-varying environment that will then influence the agent's action selection. Many studies dealing with terrestrial robot navigation [5] , spacecraft proximity and rendezvous operations [6], and self-assembly structures in space [7] have taken the approach of defining an artificial potential field to model the environment. With this method the action selection is made by following the local gradient of the artificial potential field. Although this method allows a precise modeling of the external environment, it also introduces local undesired equilibrium configurations that the system may reach. A Laplacian-based potential field [8] or the use of harmonic functions [9], along with the introduction of random walks [10], have been proposed to alleviate the problem.

Another approach to the action selection problem was introduced by Schoner and Dose [11], based on the dynamic systems theory. In this approach the state space contains behavioral variables such as heading directions or velocities. All the contributions given by each behavior are combined by means of weighting parameters into a final dynamical system that defines the course of behaviors that each agent will follow. The weighting parameters can be evaluated by solving a competitive dynamic system operating on a faster time scale. Recently other approaches have also been proposed for space applications, in the attempt to obtain some degree of decentralized coordination in a group of satellites. Ren and Beard [12] and Lawton et al. [13] introduced what they call a virtual structure method to design a decentralized formation control scheme, whereas Campbell [14] applied some results of the optimal control theory to design a coordinated formation reconfiguration maneuver. These methods aim at reaching a unique final configuration in which each satellite has its position preassigned. When a swarm of homogeneous agents is considered and the task is given to acquire a certain final geometry, the final positions occupied by each agent in the target configurations should be chosen in an autonomous way and should be part of the global behavior emerging from the individual tasks assigned.

In this paper we investigate the possibility of using the limited local sensing capabilities of each single spacecraft to coordinate the 
individual responses and achieve a common task. In particular we assume that each spacecraft can process locally sensed attitude and interspacecraft distances, to obtain a three-dimensional relative position measurement with respect to other spacecraft in the formation. The common task we try to achieve is the acquisition of a given relative geometry in which positions are not preassigned to particular satellites. We develop a behavior-based path-planning algorithm [15] able to achieve this. Based on selective sensory information, each behavior contributes to the final decision taken by the spacecraft control system. With respect to previously developed techniques the new approach presented has two advantages: it limits intersatellite communications to a minimum amount, and it autonomously assigns the satellites' positions in the final formation.

\section{Methodology}

Consider the relative motion of $N$ spacecraft, randomly distributed in the space neighboring $N$ targets, and subject to the gravitational attraction of a near planet. In the local horizontal local vertical (LHLV) reference frame associated with a given target orbit we define the target positions $\boldsymbol{\xi}_{i}, i=1 \ldots N$ and the initial states $\boldsymbol{x}_{i}^{0}, \boldsymbol{v}_{i}^{0}$, $i=1 \ldots N$ of each spacecraft. Our goal is to build a real-time navigation scheme, allowing each agent to decide its final target relying just upon its limited sensor information, and to safely navigate to it without conflicting with the other spacecraft. We use a two-step approach:

1) First, a method is developed that defines, for each target disposition and each agent neighborhood configuration, the desired velocity vector of the agent as a sum of different weighted contributions named "behaviors."

2) Next, several control techniques are considered that allow each spacecraft to track the desired kinematical field.

In this way the control design is completely independent of the design of the desired velocity field and may be tackled separately.

\section{Design of the Underlying Kinematical Field: The Equilibrium Shaping}

The approach we propose and that we call "equilibrium shaping" draws inspiration from past published works on robot path planning and artificial intelligence. In the work by Gazi [16] and Gazi and Passino [17], some theoretical results have been introduced on the dynamics of aggregating a swarm of robots. Each agent of the swarm is there asked to follow a certain velocity field defined as the sum of two different contributions, both dependent on the interagent distance $\boldsymbol{x}_{i j}=\boldsymbol{x}_{j}-\boldsymbol{x}_{i}$. The first contribution defines a linear global gather behavior whereas the second one introduces an avoidance behavior. The mathematical definition used by Gazi for the desired velocity of the $i$ th agent is

$$
\boldsymbol{v}_{d_{i}}=-\sum_{j} \boldsymbol{x}_{i j}\left[c_{i}-b_{i} \exp \left(-\frac{\boldsymbol{x}_{i j} \cdot \boldsymbol{x}_{i j}}{k_{1}}\right)\right]
$$

where $c_{i}, b_{i}$ are coefficients whose values are uniquely determined by the formation geometry, and $k_{1}$ is a parameter that determines the size of the avoid behavior sphere of influence. This method produces a swarm in which each agent is preassigned to a particular place in the final formation. We show how it is possible to achieve a global swarm behavior that also solves the target assignment problem autonomously. For a given set of positions in the space the target assignment problem is the problem of associating every single agent belonging to the swarm with every element of the set on a one-to-one basis. Such a problem can be autonomously solved by the swarm defining the desired kinematical field according to the equilibrium shaping approach proposed in this paper. This technique consists of building a dynamical system that has as equilibrium points all the possible agent permutations in the final target formation. The system is then used as a definition for the desired velocities. It is essentially an inverse approach that starts from the geometry of the final relative configuration and works out the necessary agent behaviors to reach that configuration.
Let us consider the simple example of a swarm of two satellites that aims to reach a final configuration made up of the two geometric positions given by $\xi_{1}=[1,0,0]$ and $\xi_{2}=[-1,0,0]$. We have to build a dynamical system that admits two equilibrium configurations, one in which agent 1 is in $\xi_{1}$ and agent 2 in $\xi_{2}$, and one in which the final positions are reversed.

We design our dynamic as a sum of three different behaviors that we name "gather," "avoid," and "dock." The mathematical expression of each kind of behavior along with some brief comments are listed as follows:

1) Gather Behavior. This behavior introduces $N$ different global attractors toward the $N$ targets. The analytical expression of this behavior contribution to the $i$ th agent desired velocity may be written in the following form:

$$
\boldsymbol{v}_{i}^{\text {Gather }}=\sum_{j} c_{j} \psi_{G}\left(\left\|\boldsymbol{\xi}_{j}-\boldsymbol{x}_{i}\right\|\right)\left(\boldsymbol{\xi}_{j}-\boldsymbol{x}_{i}\right)
$$

where $\psi_{G}$ is a mapping from positive real numbers to positive real numbers that introduces some nonlinear dependency from the target distance. This behavior may also be designed to account for the gravitational field, as we shall see in the next section. There are some important choices that we implicitly make when we choose this form of the gather behavior. By allowing the $c_{j}$ coefficient to depend solely on the targets and not on the agents, we make sure that each component of the swarm is identical to the others so that agent permutations do not change the swarm behavior. We also write the function $\psi_{G}$ as dependent only on the distance so that an isotropy of the desired velocity field around each hole is imposed. This may not be desired in some particular problems, in which case some angular dependency could be introduced. Moreover, because the main objective of this contribution is to gather the spacecraft from wherever they are, the $v^{\text {Gather }}$ vector is nonnegligible everywhere in the space around the target configuration.

2) Dock Behavior. This behavior introduces $N$ different local attractors toward the $N$ targets. The component of the desired velocity field due to each dock behavior has a nonnegligible value only if the agent is in the neighborhood of the sink. The $k_{D}$ parameter determines the radius of the sphere of influence of the dock behavior. The expression used for this behavior is

$$
\boldsymbol{v}_{i}^{\text {Dock }}=\sum_{j} d_{j} \psi_{D}\left(\left\|\boldsymbol{\xi}_{j}-\boldsymbol{x}_{i}\right\|, k_{D}\right)\left(\boldsymbol{\xi}_{j}-\boldsymbol{x}_{i}\right)
$$

where $\psi_{D}$ is a mapping from positive reals to positive reals that vanishes outside a given radius from the target. The same comments made for the gather behavior still apply and the dock behavior is similar to the gather one except that it is a local attractor and it therefore governs the final docking procedure. In particular the $d_{j}$ coefficients in Eq. (3) are solely dependent on the target positions and not on the agents' positions, to retain symmetry with respect to the spacecraft permutations.

3) Avoid Behavior. This behavior establishes a relationship between two different agents that are in proximity to each other. In such a case a repulsive contribution will contribute to the desired velocity field. The expression that describes the desired velocity for this kind of behavior is as follows:

$$
\boldsymbol{v}_{i}^{\text {Avoid }}=\sum_{j} b \psi_{A}\left(\left\|\boldsymbol{x}_{i}-\boldsymbol{x}_{j}\right\|, k_{A}\right)\left(\boldsymbol{x}_{i}-\boldsymbol{x}_{j}\right)
$$

where $\psi_{A}$ is a mapping from positive real to positive reals that vanishes whenever the mutual distance is considered not to be dangerous according to the value $k_{A}$. To maintain symmetry between all agents the $b$ parameter does not depend on the particular agent.

According to the definitions given before, the desired velocity field for a swarm of $N$ agents and for a final formation made of $N$ targets is defined as follows:

$$
\boldsymbol{v}_{d_{i}}=\boldsymbol{v}_{i}^{\text {Avoid }}+\boldsymbol{v}_{i}^{\text {Dock }}+\boldsymbol{v}_{i}^{\text {Gather }}
$$

The resulting dynamical system, defined by the weighted sum of 
different and often conflicting behaviors, can be written in the simple form

$$
\dot{x}=v_{d_{i}}=f(x, \lambda, \xi)
$$

where we introduce $\boldsymbol{v}_{d}=\left[\boldsymbol{v}_{d_{1}}, \ldots, \boldsymbol{v}_{d_{N}}\right], \quad \boldsymbol{x}=\left[\boldsymbol{x}_{1}, \ldots, \boldsymbol{x}_{N}\right], \quad \boldsymbol{\xi}=$ $\left[\boldsymbol{\xi}_{1}, \ldots, \boldsymbol{\xi}_{N}\right]$, and $\lambda=\left[c_{j}, d_{j}, b\right]$. This last vector contains the parameters that have to be chosen so that all the final desired configurations are equilibrium points. As we took care of retaining the symmetry of the dynamical system with respect to agent permutations, the only relation that has to be fulfilled to impose the existence of such equilibria can be written in the compact form

$$
\boldsymbol{f}(\boldsymbol{x}=\boldsymbol{\xi}, \boldsymbol{\xi}, \lambda)=0
$$

All other configurations, obtained by permutation of the $\boldsymbol{\xi}_{i}$, are granted to be also equilibrium points. This equation will be referred to as the equilibrium shaping formula, as it effectively allows us to find the value of $\lambda$ able to shape the equilibria of the dynamical system represented by Eq. (6). The study of what possible equilibria may be shaped with the preceding equation reveals to be intriguing and well described by the theory of symmetry groups. Let us take a closer look at the equilibrium shaping formula. It is a set of $N$ vectorial equations, each one related to a particular target position $\boldsymbol{\xi}_{i}$ :

$$
\begin{gathered}
\sum_{\substack{j=1 \\
i \neq j}}^{N}\left\{\left[c_{j} \psi_{G}\left(\left\|\boldsymbol{\xi}_{j}-\boldsymbol{\xi}_{i}\right\|\right)+d_{j} \psi_{D}\left(\left\|\boldsymbol{\xi}_{j}-\boldsymbol{\xi}_{i}\right\|, k_{D}\right)\right.\right. \\
\left.\left.-b \psi_{A}\left(\boldsymbol{\xi}_{j}-\boldsymbol{\xi}_{i} \|, k_{A}\right)\right]\left(\boldsymbol{\xi}_{j}-\boldsymbol{\xi}_{i}\right)\right\}=0
\end{gathered}
$$

To highlight the unknowns in Eq. (8) it is convenient write the equilibrium shaping formula in the form

$$
\boldsymbol{E}\left[c_{1}, \ldots, c_{N}, d_{1}, \ldots, d_{N}\right]^{T}=\boldsymbol{g}
$$

where the matrix $\boldsymbol{E}$ and the vector $\boldsymbol{g}$ depend on the functions $\psi$ chosen to represent the various behaviors on the target positions $\xi$ and on the parameter $b$. This set of equations represents, for each target disposition and for each choice of the parameters $k_{D}$ and $k_{A}$, a linear set of equations in the $2 N$ unknowns $c_{j}, d_{j}$. Depending on the spatial distribution of the target points we might be able to find solutions. Let us further investigate the general case: a set of $3 \mathrm{~N}$ equations in $2 N$ unknowns and no possible solution. We must rely upon the linear dependence of some of the equations. We introduce the punctual symmetry group $G$ of the target positions. $G$ contains all the matrices $\boldsymbol{R} \in \operatorname{orth}(3)$ that map the set of the target positions into itself. Whenever two target points $\boldsymbol{\xi}_{i}, \boldsymbol{\xi}_{j}$ are equivalent with respect to $G$ (i.e., $\exists \boldsymbol{R} \in G$ such that $\boldsymbol{R} \boldsymbol{\xi}_{i}=\boldsymbol{\xi}_{j}$ ) then the two corresponding equations are linearly dependent if we set $c_{i}=c_{j}, d_{i}=d_{j}$. This statement can easily be proven by premultiplying Eq. (8) by a matrix $\boldsymbol{R} \in G$ and using the identity between the various coefficients. This simple trick allows us to count the number of independent vectorial equations.

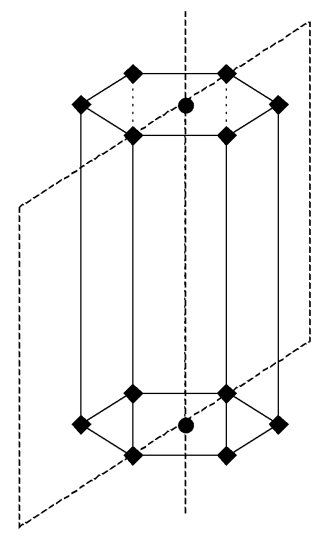

Fig. 1 Formation with a hexagonal-P shape.
Table 1 Count of the equations and the unknowns for different formations

\begin{tabular}{lcc}
\hline \hline Formation shape & Number of equations & Number of unknowns \\
\hline All regular solids & 1 & 2 \\
All regular polygons & 1 & 2 \\
Pyramids with a regular & 3 & 4 \\
$\quad$ basis & 1 & 2 \\
Cubic-P & 1 & 4 \\
Cubic-I & 2 & 4 \\
Cubic-F & 2 & 2 \\
Tetragonal-P & 2 & 4 \\
Tetragonal-I & 3 & 2 \\
Orthorhombic-P & 3 & 4 \\
Orthorhombic-I & 4 & 4 \\
Orthorhombic-C & 6 & 4 \\
Orthorhombic-F & 3 & 4 \\
Hexagonal-P & & \\
\hline \hline
\end{tabular}

Once we know how many independent vectorial equations we are dealing with, we also know that each of them counts as three, two, one, or even zero scalar independent equations according to the following simple rule: each independent vectorial equation written for the $\boldsymbol{\xi}_{i}$ position is equivalent to a single scalar equation whenever a symmetry axis passes through it, to two scalar equations if a symmetry plan passes through $\xi_{i}$, and it is an identity if more than one symmetry axis passes through $\boldsymbol{\xi}_{i}$. If none of these rules is applicable, the equation counts as three scalar independent equations.

As an example let us consider the hexagonal Bravais lattice shown in Fig. 1. The target positions belonging to this formation can be divided into two different groups (the prism vertices and the base centers) belonging to two different symmetry classes. We therefore start with two linearly independent vectorial equations. As a symmetry plane passes through the vertices, the corresponding vectorial equation counts as two equations. As a symmetry axis passes through the hexagon centers, the corresponding vectorial equation counts as one equation and the equilibrium shaping formula may therefore reduced to a total of three scalar equations if we set $c_{i}=c_{j}, d_{i}=d_{j}$ among the positions belonging to the same group.

In Table 1 some formations are listed together with the number of independent equations and unknowns that characterize them. Some of the formations presented in Table 1 draw inspiration from the wellknown Bravais lattice spatial configurations, illustrated in Fig. 2.

Whenever the number of equations is less than the number of unknowns, the choice of the various parameters may be made in many different ways. Exploiting this fact, the choice may be made

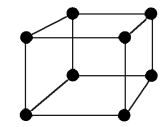

Cubic-P

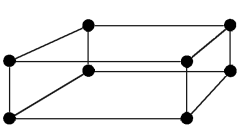

Orthorombic-P
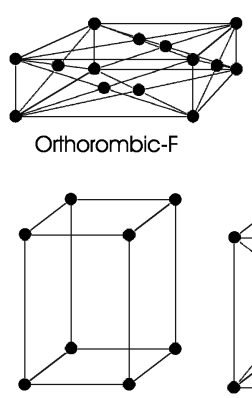

Tetragonal-P

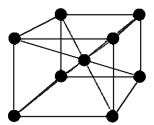

Cubic-I

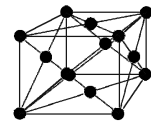

Cubic-F

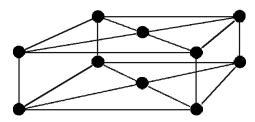

Orthorombic-C

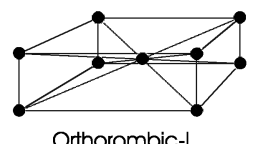

Orthorombic-1
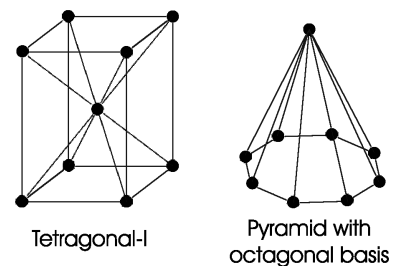

octagonal basis
Fig. 2 Visualization of some formations. 
differently by each agent and may be seen as the "subjective" view that the $i$ th spacecraft has of the equilibrium condition. There is no need to agree on the particular solution chosen.

Let us consider the case in which the target positions $\xi_{i}$ form an icosahedron. For this particular target geometry (see Table 1 under regular solids) the equilibrium shaping formula reduces to $\bar{a}$ single scalar relation:

$$
\begin{aligned}
c= & {\left[5(R-h) e^{-\ell^{2} / k_{A}}+5(R+h) e^{-\ell^{\prime 2} / k_{A}}+2 R e^{-2 R^{2} / k_{A}}\right] b } \\
& -\left[5(R-h) e^{-\ell^{2} / k_{D}}+5(R+h) e^{-\ell^{\prime 2} / k_{D}}+2 R e^{-2 R^{2} / k_{D}}\right] d
\end{aligned}
$$

where the quantities $\ell$ and $R$ are, respectively, the edges of the icosahedron and the radius of the sphere in which the icosahedron can be inscribed, whereas $\ell^{\prime}$ and $h$ can be written as follows:

$$
h=\sqrt{R-\left(\frac{\ell}{2 \sin \left(\frac{\pi}{10}\right)}\right)^{2}} \quad \ell^{\prime}=\sqrt{(R+h)^{2}+\left(\frac{\ell}{2 \sin \left(\frac{\pi}{10}\right)}\right)^{2}}
$$

For a fixed parameter $b$, Eq. (9) defines a relation between the two remaining parameters $c$ and $d$. Any choice of these two parameters leads to a dynamical system describing the desired velocity having the icosahedron as equilibrium point. Such a dynamical system has the following form:

$$
\begin{aligned}
\dot{\boldsymbol{x}}_{i} & =\sum_{j=1}^{N}\left[-b \exp \left(-\left\|\boldsymbol{x}_{i}-\boldsymbol{x}_{j}\right\|^{2} / k_{A}\right)\right]\left(\boldsymbol{x}_{i}-\boldsymbol{x}_{j}\right) \\
& +\sum_{j=1}^{N}\left[-c-d \exp \left(-\left\|\boldsymbol{\xi}_{i}-\boldsymbol{\xi}_{j}\right\|^{2} / k_{D}\right)\right]\left(\boldsymbol{\xi}_{i}-\boldsymbol{\xi}_{j}\right)
\end{aligned}
$$

The expression used for the function $\psi$ is the one proposed by Gazi [16] and Gazi and Passino [17]. The function $\psi$ may be defined in different ways to decrease the computational load as done, for example, by Large et al. [18] where a simple sine function is used. One important issue that has to be taken into account in the definition of the $\psi_{A}$ function is that of collision avoidance. In particular a proper definition of the $\psi_{A}$ function has to take into account the real dimensions of the spacecraft belonging to the formation so that collision between the spacecraft can be avoided with a reasonable margin. In Fig. 3 the outcome of a numerical integration of the dynamical system defined by Eq. (6) is shown in the case $b=1.1 \mathrm{~s}^{-1}, d=0.3 \mathrm{~s}^{-1}, k_{1}=k_{2}=1 \mathrm{~m}^{2}, l=2 \mathrm{~m}$. The initial positions of the various agents have been randomly generated on the surface of a sphere of radius 20 times the length of the icosahedron

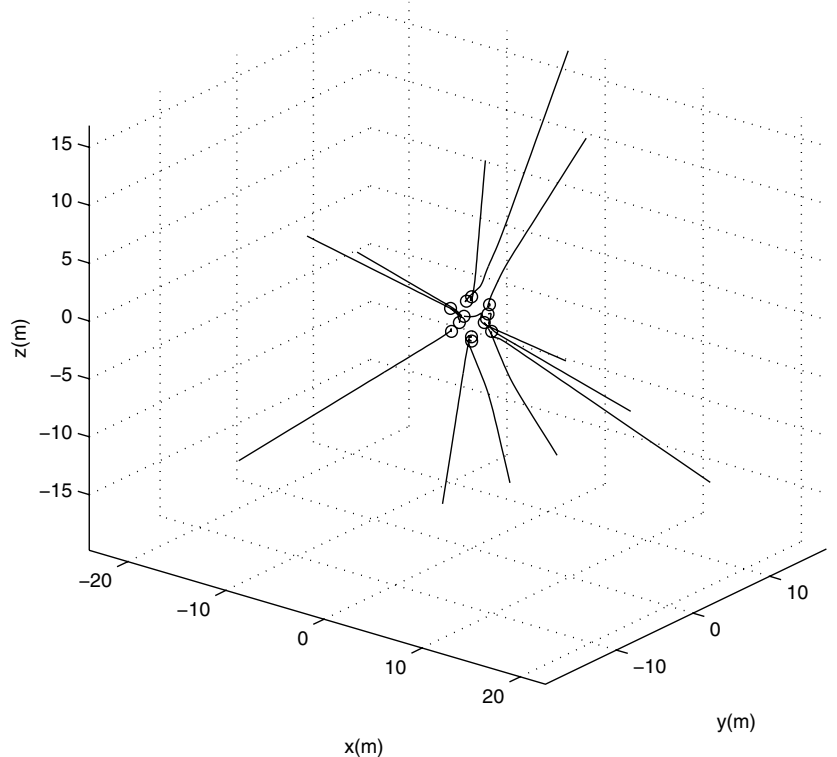

Fig. 3 Example of desired trajectories for an icosahedron-shaped target formation. edge. In this particular simulation the minimum distance occurring between two spacecraft is 0.87 times the length of the icosahedron edge. The lines shown in Fig. 3 are the trajectories that each agent, having chosen $d$ and $c$, foresees and uses to evaluate its desired velocity. We note that the agents do not perform any numerical integration to plan their path, only a simple algebraic calculation. During various simulations the rise of emerging behaviors due to the interaction between different conflicting behaviors [15] may be observed. As a final remark we note here that using the functions $\psi$ proposed by Gazi [16] may lead to local minima configurations, that is, equilibrium configurations different from the desired one. A proper choice of the $b, c$, and $d$ parameters in the space of the solutions of the equilibrium shaping formula significantly alleviates this problem [19].

\section{Exploiting the Gravitational Environment}

The desired kinematical field designed in the preceding section allows the final formation to be reached following forced trajectories that are not geodesics. It is not difficult to imagine that the control system will struggle to follow these trajectories, using unnecessarily large amount of propellant whenever the gravitational forces become significant. A modification may be introduced that takes into account and exploits the geodesics, to reduce the overall mass consumption. We start from the well-known system of Hill equations [20]:

$$
\left\{\begin{array}{l}
\ddot{x}-2 \omega \dot{y}-3 \omega^{2} x=0 \\
\ddot{y}+2 \omega \dot{x}=0 \\
\ddot{z}+\omega^{2} z=0
\end{array}\right.
$$

where $\rho=[x, y, z]$ is the position of the spacecraft with respect to an LHLV reference frame moving with angular velocity $\omega$ (the reference orbit is here circular). This set of equations has an exact analytical solution in the form

$$
\left[\begin{array}{c}
\rho \\
\dot{\rho}
\end{array}\right]=\left[\begin{array}{ll}
A(t) & B(t) \\
C(t) & D(t)
\end{array}\right]\left[\begin{array}{c}
\rho_{0} \\
\dot{\rho}_{0}
\end{array}\right]
$$

where $t$ is time, $\left[\boldsymbol{\rho}_{0}, \dot{\boldsymbol{\rho}}_{0}\right]=[\boldsymbol{\rho}(t=0), \dot{\boldsymbol{\rho}}(t=0)]$, and the matrices $\boldsymbol{A}$, $\boldsymbol{B}, \boldsymbol{C}$, and $\boldsymbol{D}$ can be easily found in the literature [7]. The preceding solution can be used to define a new gather behavior that exploits the gravitational force to reach the final desired configuration. Requiring that a given satellite has to reach a certain point $\rho_{d}$ after a fixed time $t_{d}$, the following relation has to be fulfilled:

$$
\rho_{d}=\rho\left(t_{d}\right)=\boldsymbol{A}\left(t_{d}\right) \boldsymbol{x}_{0}+\boldsymbol{B}\left(t_{d}\right) \dot{\boldsymbol{x}}_{0}
$$

Taking this into account we may assign for each position in space $\boldsymbol{x}_{i}$ and each target belonging to the final formation $\boldsymbol{\xi}_{j}$ a new gather velocity vector given by

$$
\boldsymbol{v}_{i}^{\text {Gather }}=\frac{1}{N} \sum_{j} \boldsymbol{B}^{-1} \boldsymbol{\xi}_{j}-\boldsymbol{B}^{-1} \boldsymbol{A} \boldsymbol{x}_{i}
$$

where $\boldsymbol{A}=\boldsymbol{A}\left(\hat{\tau}_{d}-t\right), \boldsymbol{B}=\boldsymbol{B}\left(\hat{\tau}_{d}-t\right)$, and $\hat{\tau}_{d}$ is the time in which, from the beginning of the simulation, the agent is required to reach the center of the desired formation. Note that no boundary condition can be enforced on the final velocity of the approach phase as long as the parameter $\hat{\tau}_{d}$ is held fixed. Even though the resulting desired velocity vector depends explicitly on the time, in a practical application the agent clocks need not be synchronized. This contribution is added to the dock behavior and the avoid behavior to build the final desired kinematical field. Unfortunately Eq. (12) is singular when $t$ approaches $\hat{\tau}_{d}$, that is, in the final part of the target acquisition. Besides, near the targets the desired velocity due to this new gather behavior is higher than needed (the spacecraft has to get out of a ballistic trajectory to acquire the targets). For these reasons the desired kinematical field will be divided in two different parts, one far from the desired final configuration, in which the gather behavior takes into account the gravitational force, and one close to the desired final formation, in which space is considered to be flat. 
The geometrical shape of the edges of these two different zones of space can be easily set as a sphere of radius $R_{s}$, that can be considered, together with the desired gather time $\hat{\tau}_{d}$, as a parameter to be decided by the system designer.

\section{Feedback Synthesis}

In general the agent will not possess the desired velocity and a control system has to be designed that is able to reduce the error between the actual velocity and the desired one. In this section, different feedbacks achieving this will be derived and discussed. A simplified dynamics is considered throughout this chapter for the control design purpose. In particular, the motion of the $i$ th satellite belonging to the swarm is modeled according to Eq. (11). The performances of the derived feedback schemes are then tested in the next sections with a full nonlinear simulation.

\section{A. Q-Guidance}

The first feedback we develop is inspired by the Q-guidance steering law formally introduced by Battin [21] for rocket guidance. It is based on definition of the velocity-to-be-gained vector $\boldsymbol{v}_{g_{i}}$ that represents, in our case, the instantaneous difference between each agent's actual velocity $\boldsymbol{v}_{i}$ and desired velocity $\boldsymbol{v}_{d_{i}}$. The objective of the control system is to drive the velocity-to-be-gained vector to zero. From now on each quantity will be related to one agent. To simplify the notation the subscript identifying the agent will be omitted. We define the following function:

$$
V=\frac{1}{2} \boldsymbol{v}_{g} \cdot \boldsymbol{v}_{g}
$$

The velocity-to-be-gained vector decreases along the trajectories followed by each agent if and only if

$$
\dot{V}=\boldsymbol{v}_{g} \cdot \dot{\boldsymbol{v}}_{g}<0
$$

The time derivative of $\boldsymbol{v}_{g}$ during the motion has the expression

$$
\dot{\boldsymbol{v}}_{g}=\dot{\boldsymbol{v}}_{d}-\dot{\boldsymbol{v}}
$$

We substitute into this relation the momentum balance of each spacecraft written in the LHLV frame:

$$
\dot{v}=a_{\text {in }}+a_{\mathrm{dis}}+\boldsymbol{u}
$$

where $\boldsymbol{u}$ is the control vector, $\boldsymbol{a}_{\text {in }}$ is the acceleration of the spacecraft due to inertial forces, and $\boldsymbol{a}_{\mathrm{dis}}$ is the acceleration due to nonmodeled disturbance forces. The following expression is obtained:

$$
\dot{\boldsymbol{v}}_{g}=\dot{\boldsymbol{v}}_{d}-\boldsymbol{a}_{\mathrm{in}}-\boldsymbol{a}_{\mathrm{dis}}-\boldsymbol{u}
$$

Because $\dot{\boldsymbol{v}}_{d}$ is a sole function of $t$ and $\boldsymbol{x}$ [see Eqs. (2-5) and (12)], we may express the desired velocity derivative in the following form:

$$
\dot{\boldsymbol{v}}_{d}=\frac{\partial \boldsymbol{v}_{d}}{\partial t}+\frac{\partial \boldsymbol{v}_{d}}{\partial \boldsymbol{x}} \boldsymbol{v}=\frac{\partial \boldsymbol{v}_{d}}{\partial t}+\frac{\partial \boldsymbol{v}_{d}}{\partial \boldsymbol{x}}\left(\boldsymbol{v}_{d}-\boldsymbol{v}_{g}\right)
$$

As an example we consider the contribution given by the sole gather behavior defined by Eq. (12). In this particular case the time derivative of the desired velocity has the expression

$$
\dot{\boldsymbol{v}}_{d}=\boldsymbol{a}_{\mathrm{in}}-\frac{\partial \boldsymbol{v}_{d}}{\partial \boldsymbol{x}} \boldsymbol{v}_{g}
$$

and the resulting total time derivative of the velocity to be gained may be written in the following compact form:

$$
\dot{\boldsymbol{v}}_{g}=-\boldsymbol{u}-\boldsymbol{a}_{\mathrm{dis}}-\frac{\partial \boldsymbol{v}_{d}}{\partial \boldsymbol{x}} \boldsymbol{v}_{g}=-\boldsymbol{u}-\boldsymbol{a}_{\mathrm{dis}}-\boldsymbol{B}^{-1} \boldsymbol{A} \boldsymbol{v}_{g}
$$

To make $\dot{V}<0$ we introduce the following feedback:

$$
\boldsymbol{u}=\kappa \boldsymbol{v}_{g}+\dot{\boldsymbol{v}}_{d}-\boldsymbol{a}_{\text {in }}
$$

in which $\kappa>0$ is a positive real parameter whose choice will be discussed later in this section. Substituting Eq. (15) in Eq. (13) we have

$$
\dot{V}=\boldsymbol{v}_{g} \cdot\left(-\kappa \boldsymbol{v}_{g}-\boldsymbol{a}_{\mathrm{dis}}\right)=-\kappa\left\|\boldsymbol{v}_{g}\right\|^{2}-\boldsymbol{a}_{\mathrm{dis}} \cdot \boldsymbol{v}_{g}<0
$$

Note that the Lyapunov function in Eq. (16) contains an uncertain term that does not allow, in principle, any conclusion on the convergence of the feedback. If $\left\|\boldsymbol{a}_{\text {dis }}\right\|<\bar{a}_{\text {dis }}$ it is, however, possible to find a lower bound on the value of the parameter $\kappa$ that ensures $v_{g}<\tilde{v}_{g}$. For a given $\boldsymbol{v}_{g}$ the worst scenario occurs whenever the unknown perturbation is such that

$$
\boldsymbol{a}_{\mathrm{dis}}=-\bar{a}_{\mathrm{dis}} \frac{\boldsymbol{v}_{g}}{\left\|\boldsymbol{v}_{g}\right\|}
$$

Therefore if $\kappa>\bar{a}_{\text {dis }} / \tilde{v}_{g}$, it follows that $\dot{V}<0$ along the trajectories followed by each agent as long as $\left\|\boldsymbol{v}_{g}\right\|>\tilde{v}_{g}$.

In real cases the magnitude of the control feedback cannot be arbitrarily large because the agents will typically have an upper limit to the thrust magnitude. Therefore a saturation level must be introduced into the feedback defined in Eq. (15). This way we lose the mathematical result on the feedback global stability. Nevertheless, it is always possible to choose the $\psi$ functions and parameters $b, k_{A}$, and $k_{D}$ such that local stability is ensured, that is, for limited $\left\|\boldsymbol{v}_{g}(t=0)\right\|$ the saturation level is never reached.

Different choices of the $\kappa$ parameter in Eq. (15) allow derivation of different feedback strategies. In particular, steering laws analogous to the ones based on the Q-guidance [21] approach can be obtained. In Fig. 4 the vectorial diagram of Eq. (15) is shown for two different cases. $\overline{\boldsymbol{u}}$ represents the control vector constrained in magnitude to $u_{\text {sat }}$. The dotted line in both subfigures $4 \mathrm{a}$ and $4 \mathrm{~b}$ represents the direction of the velocity-to-be-gained vector at any given time.

If $\kappa \rightarrow \infty$, the control strategy is to thrust in the direction of the velocity-to-be-gained vector as shown in Fig. $\underline{4 a}$, regardless of the contribution to $\dot{\boldsymbol{v}}_{g}$ due to the noncontrollable terms $\dot{\boldsymbol{v}}_{d}$ and $\boldsymbol{a}_{\text {in }}$. A different strategy is to try aligning the time derivative of $\boldsymbol{v}_{g}$ to $\boldsymbol{v}_{g}$ itself (as shown Fig. 4 b). In this case

$$
\dot{\boldsymbol{v}}_{g} \times \boldsymbol{v}_{g}=0
$$

We obtain the known cross-product steering law [21]. In our notation, the corresponding value of $\kappa$ is such that

$$
\left(\kappa \boldsymbol{v}_{g}+\dot{\boldsymbol{v}}_{d}-\boldsymbol{a}_{\mathrm{in}}\right) \cdot\left(\kappa \boldsymbol{v}_{g}+\dot{\boldsymbol{v}}_{d}-\boldsymbol{a}_{\mathrm{in}}\right)=u_{\mathrm{sat}}^{2}
$$

where $u_{\text {sat }}$ is the saturation considered for the thrust vector modulus.
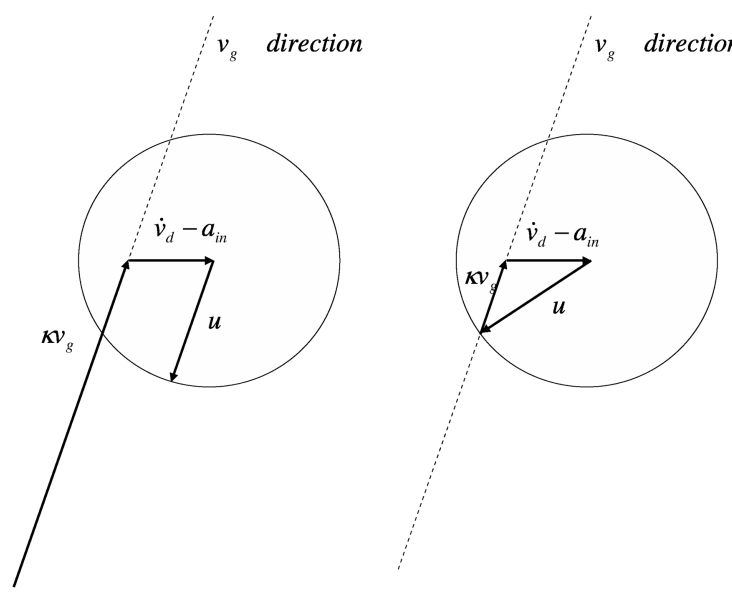

a)

b) 


\section{B. Sliding-Mode Control}

A state feedback can also be obtained using the results of the sliding-mode control theory [22]. The aim of this approach is to design a control law able to drive the system trajectory on a predetermined manifold and keep it there once reached. The control design procedure can be broken down in two different steps: first we design a sliding manifold (or switching manifold) such that the system motion restricted to it leads the swarm of satellites towards the desired equilibrium configuration. Then we derive a control law that forces the trajectories of the system to converge to the sliding manifold and to stay on it thereafter.

The dynamical system to be controlled for each agent is

$$
\left\{\begin{array}{l}
\dot{\boldsymbol{v}}_{i}=\boldsymbol{a}_{\mathrm{in}_{i}}+\boldsymbol{a}_{\mathrm{dis}_{i}}+\boldsymbol{u}_{\mathrm{i}} \\
\dot{\boldsymbol{x}}_{i}=\boldsymbol{v}_{i}
\end{array}\right.
$$

where $\boldsymbol{a}_{\mathrm{in}_{i}}$ is the inertial acceleration acting upon the agent; $\boldsymbol{a}_{\mathrm{dis}_{i}}$ is the disturbance acceleration such that $\left\|\boldsymbol{a}_{\mathrm{dis}}\right\|<\bar{a}_{\mathrm{dis}_{i}}$, and $\boldsymbol{u}_{i}$ is the control vector. Following the notation already introduced in this section for the sake of simplicity, the index will be omitted hereafter. The sliding manifold may be written in the following form:

$$
\sigma(x, v, t)=0
$$

where the relation $\sigma(\boldsymbol{x}, \boldsymbol{v}, t)$ has to be chosen such that the trajectory that the system follows by "sliding" on the manifold will cause the system to reach the final desired formation. The equilibrium shaping technique we introduced in this paper is in fact a method to build such a sliding manifold. For the system in Eq. (19) the expression we use for $\sigma(x, v, t)$ is

$$
\boldsymbol{\sigma}(\boldsymbol{x}, \boldsymbol{v}, t)=\boldsymbol{v}_{d}-\boldsymbol{v}=0
$$

where $\boldsymbol{v}_{d}$ is defined in Eq. (5). Whenever the system is on the sliding manifold it will stay on it if and only if the following relation is satisfied at all times:

$$
\dot{\sigma}(x, v, t)=0
$$

That is, according to Eq. (19) and to Eq. (20)

$$
\dot{\boldsymbol{v}}_{d}-\dot{\boldsymbol{v}}=\dot{\boldsymbol{v}}_{d}-\boldsymbol{a}_{\mathrm{in}}-\boldsymbol{a}_{\mathrm{dis}}-\boldsymbol{u}=0
$$

It is then possible to define the equivalent control $\boldsymbol{u}_{\text {eq }}$ as a feedback that keeps the state of the nominal system on the manifold at all times:

$$
\boldsymbol{u}_{\mathrm{eq}}=-\boldsymbol{a}_{\text {in }}+\dot{\boldsymbol{v}}_{d}
$$

A particular case is when the gravitational gather behavior is the only contribution to the desired velocity. Then the control force reduces to zero, because on the sliding manifold we have

$$
\dot{\boldsymbol{v}}_{d}=\frac{\partial \boldsymbol{v}_{d}}{\partial t}+\frac{\partial \boldsymbol{v}_{d}}{\partial \boldsymbol{x}} \boldsymbol{v}_{d}=\boldsymbol{a}_{\mathrm{in}}
$$

The dynamical system in Eq. (19) subject to the equivalent control $\boldsymbol{u}_{\text {eq }}$ will never leave the sliding manifold after having intersected it once. It is now necessary to add to the equivalent control another term that acts when $\sigma \neq 0$ and is able to drive the system trajectory to intersect the sliding manifold. The total control vector can then be expressed as the sum of two contributions:

$$
\boldsymbol{u}=\boldsymbol{u}_{\mathrm{eq}}+\boldsymbol{u}_{N}
$$

where $\boldsymbol{u}_{\text {eq }}$ has been defined in Eq. (21). The vector $\boldsymbol{u}$ applied to the system Eq. (19) couples the dynamics of each single spacecraft to the other components of the swarm that the spacecraft can sense. A switched control law in the form

$$
u_{N_{i}}=\left\{\begin{array}{l}
u_{N_{i}}^{+}(\boldsymbol{x}, \boldsymbol{v}, t) \longleftrightarrow \sigma_{i}(\boldsymbol{x}, \boldsymbol{v}, t)>0 \\
u_{N_{i}}^{-}(\boldsymbol{x}, \boldsymbol{v}, t) \longleftrightarrow \sigma_{i}(\boldsymbol{x}, \boldsymbol{v}, t)<0
\end{array}\right.
$$

will force the system to fall onto the sliding manifold if the values
$u_{N_{i}}^{+}, u_{N_{i}}^{-}$are chosen so that the velocities of the system will always point towards it. The value chosen for the feedback gains is determined according to the sign of the components of $\boldsymbol{\sigma}(\boldsymbol{x}, \boldsymbol{v}, t)$, also called the "switching surface" for this reason. The control $u_{N_{i}}$ is set to zero on the switching surface. A Lyapunov method can be used to find the values of the switching gains. Let us define the following Lyapunov function:

$$
V=\frac{1}{2} \sigma \cdot \sigma
$$

Then a control feedback must be derived to impose the time derivative of $V$ to be definite negative along the trajectory of the system. The condition on the total time derivative of the Lyapunov function can be imposed by

$$
\dot{V}=\left(\boldsymbol{v}_{d}-\boldsymbol{v}\right) \cdot\left(\dot{\boldsymbol{v}}_{d}-\dot{\boldsymbol{v}}\right)<0
$$

Recalling Eqs. (21) and (22), this becomes

$$
\dot{V}<0 \Longleftrightarrow\left(v_{d}-v\right) \cdot\left(\boldsymbol{u}_{N}-\boldsymbol{a}_{\mathrm{dis}}\right)>0
$$

The latter equation can be written in terms of the velocity to be gained vector already defined:

$$
\boldsymbol{v}_{g} \cdot\left(\boldsymbol{u}_{N}-\boldsymbol{a}_{\mathrm{dis}}\right)>0
$$

Each additional feedback law $\boldsymbol{u}_{N}$ that meets this condition can be used to drive the motion of the system towards the sliding manifold. Consistently with the work presented by Gazi [16] and to keep the derivation close to the classical sliding-mode approach, the so-called relays with constant gain [22] thrusting strategy for $\boldsymbol{u}_{N}$ is introduced:

$$
\boldsymbol{u}_{N}=u_{0} \operatorname{sign}(\boldsymbol{\sigma})=u_{0} \operatorname{sign}\left(\boldsymbol{v}_{g}\right)
$$

where the sign function is defined componentwise and $u_{0}>\bar{a}_{\mathrm{dis}}$. This definition clearly satisfies Eq. (23) and leads the system to reach the sliding manifold and then the desired equilibrium configuration. In real applications it is not usually possible to achieve ideal sliding mode with the feedback $\boldsymbol{u}_{N}$, due to the occurrence of the so-called chattering phenomena [16]. Different techniques may be considered to mitigate this effect such as the boundary layer controller [23]. A discussion about these methods is not included here for reasons of space. As a final remark we note that the thrusting strategy $\boldsymbol{u}=$ $\kappa \boldsymbol{v}_{g}+\dot{\boldsymbol{v}}_{d}-\boldsymbol{a}_{\text {in }}$ inspired by the Q-guidance method can be also written as $\boldsymbol{u}=\boldsymbol{u}_{\mathrm{eq}}+\boldsymbol{u}_{N}$, where $\boldsymbol{u}_{N}=\kappa \boldsymbol{v}_{g}$. In this sense, with the sliding-mode theory one is also able to derive the feedback obtained by following the velocity-to-be-gained vector approach.

\section{Artificial Potential Approach}

A different thrusting strategy can be obtained by starting from the definition of an artificial potential function [6] for the whole swarm $V\left(\boldsymbol{x}_{1}, \cdots, \boldsymbol{x}_{n}, \boldsymbol{v}_{1}, \cdots, \boldsymbol{v}_{n}\right)$, which has minimum points at all the possible agent permutations in the final desired formation. Such a function of the state of the system can be written as

$$
\begin{aligned}
V & =\frac{1}{2} \sum_{i} \boldsymbol{v}_{i} \cdot \boldsymbol{v}_{i}+\sum_{i} \sum_{j \neq i} \phi_{A}^{i j}\left(\boldsymbol{x}_{i}-\boldsymbol{x}_{j}\right)+\sum_{i} \sum_{j} \phi_{G}^{i j}\left(\boldsymbol{x}_{i}-\boldsymbol{\xi}_{j}\right) \\
& +\sum_{i} \sum_{j} \phi_{D}^{i j}\left(\boldsymbol{x}_{i}-\boldsymbol{\xi}_{j}\right)
\end{aligned}
$$

where $\phi_{A}^{i j}, \phi_{D}^{i j}$, and $\phi_{G}^{i j}$ are defined according to the equilibrium shaping technique so that

$$
\frac{\partial \phi_{A}^{i j}}{\partial \boldsymbol{x}_{i}}=-\boldsymbol{v}_{i}^{\text {Avoid }} \quad \frac{\partial \phi_{G}^{i j}}{\partial \boldsymbol{x}_{i}}=-\boldsymbol{v}_{i}^{\text {Gather }} \quad \frac{\partial \phi_{D}^{i j}}{\partial \boldsymbol{x}_{i}}=-\boldsymbol{v}_{i}^{\text {Dock }}
$$

and each quantity labeled with the $i$ index is related to the $i$ th agent. The swarm will reach the target formation, avoiding intervehicle collisions, whenever the function $V\left(\boldsymbol{x}_{1}, \cdots, \boldsymbol{x}_{n}, \boldsymbol{v}_{1}, \cdots, \boldsymbol{v}_{n}\right)$ strictly decreases during the motion. We get 


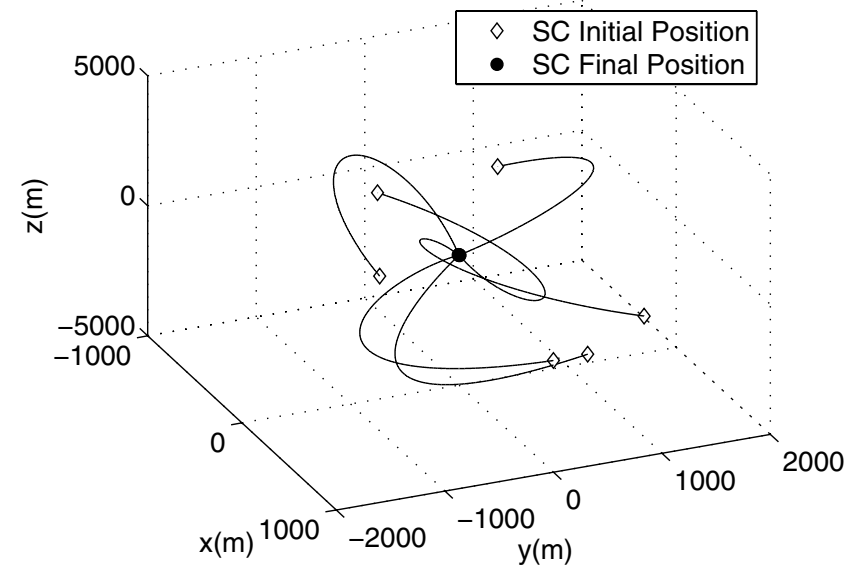

Fig. 5 Simulation of a swarm of satellites reaching a hexagonal regular formation. First approaching phase.

$$
\dot{V}=\sum_{i}\left(\frac{\partial V}{\partial \boldsymbol{x}_{i}} \dot{\boldsymbol{x}}_{i}+\frac{\partial V}{\partial \boldsymbol{v}_{i}} \dot{\boldsymbol{v}}_{i}\right)=\sum_{i}\left(\dot{\boldsymbol{v}}_{i}-\boldsymbol{v}_{d_{i}}\right) \cdot \boldsymbol{v}_{i}<0
$$

Taking into account the $i$ th agent's equation of motion, it is possible to use the following feedback $\boldsymbol{u}_{i}=\boldsymbol{v}_{d_{i}}-\kappa_{i} \boldsymbol{v}_{i}-\boldsymbol{a}_{\mathrm{in}_{i}}$. Written in terms of the velocity-to-be-gained vector, this becomes

$$
\boldsymbol{u}_{i}=\kappa_{i} \boldsymbol{v}_{g_{i}}+\left(1-\kappa_{i}\right) \boldsymbol{v}_{d_{i}}-\boldsymbol{a}_{\mathrm{in}_{i}}
$$

With this feedback the time derivative of the potential function is

$$
\dot{V}\left(x_{1}, \cdots, x_{n}, v_{1}, \cdots, v_{n}\right)=\sum_{i} v_{i} \cdot\left(-\kappa_{i} v_{i}+a_{\mathrm{dis}}\right)
$$

which can be made definite negative for a certain range of spacecraft velocities, so long as lower bounds on the $\kappa_{i}$ parameters are chosen using the same procedure described for the Q-guidance feedback. With respect to the previously presented feedback design methods, the one showed here relies upon a slightly different approach. First a global artificial potential function is defined for the entire swarm of satellites. This function is required to have a minimum value in correspondance of the target formation (permutations are allowed) and this may be achieved using the equilibrium shaping approach. Next a control law is imposed, such that the potential function decreases along the trajectories followed by the system. The resulting feedback cannot be obtained from the Q-guidance or the sliding mode and therefore represents an alternative to be considered.

\section{Simulation Results}

In this section we present some numerical simulations performed to study the performances of the discussed behavior-based control in a geostationary (GEO) orbital environment. The simulation integrates the equation of motion of each spacecraft, written in an inertial frame, in which a disturbance acceleration has been included to capture the perturbations due to differential solar radiation drag and gravitational zonal harmonics, and modeled as white noise of equivalent amplitude. We randomly placed $N$ satellites within a certain range and we activated the controller to study the swarm behavior. We performed our simulations for different relative geometries. We also considered different feedbacks given by the following:

1) Equation (15) with $\kappa$ such that Eq. (18) is satisfied. We called this feedback cross-product steering law (CPSL).

2) Equation (22) with $u_{0}$ tuned in such a way as to make the acquisition time of the final targets equal to that of the CPSL case. We called this feedback sliding-mode control (SMC).

3 ) Equation (15) with $\kappa$ tuned as for the SMC. We called this feedback velocity to be gained (VTBG).

4) Equation (26) with $\kappa$ tuned as for the SMC. We called this feedback artificial potential feedback (APF).
Typically, as a consequence of the control actuation, each agent path includes different phases:

1) A powered part in which the initial velocity-to-be-gained vector is driven to zero and in which a ballistic trajectory is reached.

2) A coasting phase in which the desired velocity and the actual velocity are identical and the control system does not use the actuators.

3) A last phase, activated within the sphere of radius $R_{s}$, in which the agents are near to the final targets and each navigates toward one of them, and the final geometry is acquired. In this phase the gather behavior did not take into account the gravitational effect.

The final relative geometry was achieved only when every agent occupied a target position such that the equilibrium shaping formula was satisfied and the desired velocities were all zero. As an example we show the trajectories followed by six satellites achieving a hexagonal formation with a $6 \mathrm{~m}$ radius. For a hexagonal formation the equilibrium shaping formula reduces to a single equation in two unknowns, $c$ and $d$. In a geostationary environment the dominating perturbation is due to the solar radiation pressure [24]. For highaltitude orbits (from $2.5 \times 10^{4} \mathrm{~km}$ ) this perturbation is in fact 1 order of magnitude larger then that induced by the higher harmonics of the Earth's gravity field, and to that induced by air drag. For a cylindrical spacecraft of mass $50 \mathrm{~kg}$, radius $0.5 \mathrm{~m}$, and height $1.5 \mathrm{~m}$, a worst-case value for the differential solar radiation drag is about $4 \times 10^{-7} \mathrm{~m} / \mathrm{s}^{2}$. In the simulation a random acceleration of magnitude $10^{-6} \mathrm{~m} / \mathrm{s}^{2}$ was included to model this effect with a conservative margin. Each spacecraft belonging to the swarm started an average distance of $1000 \mathrm{~m}$ from the center of the final configuration. The saturation value used for the thrust acceleration modulus was $0.005 \mathrm{~m} / \mathrm{s}^{2}$ and the feedback law used is the VTBG. The final formation was achieved after $40,000 \mathrm{~s}$, corresponding to roughly 0.5 times the reference orbital period. This result was obtained by setting $b=0.1222 \mathrm{~s}^{-1}, d=0.0444 \mathrm{~s}^{-1}$, and $k_{A}=k_{D}=3 \mathrm{~m}$. The corresponding value of the $c$ parameter calculated according to the equilibrium shaping formula was $c=2.2656 \times 10^{-4} \mathrm{~s}^{-1}$. The parameter $\hat{\tau}_{d}$ appearing in the modified gather behavior was set to $\hat{\tau}_{d}=39,000 \mathrm{~s}$. The choice of all such parameters, together with the selection of the $\psi$ functions appearing in Eqs. (2-4), is a crucial issue, and it deeply affects the properties of the desired kinematical field. In particular relatively high values of the $b$ parameter with respect to $c$ and $d$ allow reduction of the probability of collisions between the spacecraft. On the other hand, a high value of the $c$ parameter reduces the time required to acquire the target configuration. Therefore the choice of such parameters always results from a tradeoff between different objectives and may change from case to case. In Figs. $\underline{5}$ and 6 , the trajectories followed by the spacecraft belonging to the swarm are displayed. Figure 5 shows the motion of the swarm in the outer part of the kinematical field where the gather behavior is given by Eq. (12), whereas Fig. 6 shows the motion of the swarm in the very last phase where the gather behavior is given by Eq. (2). The

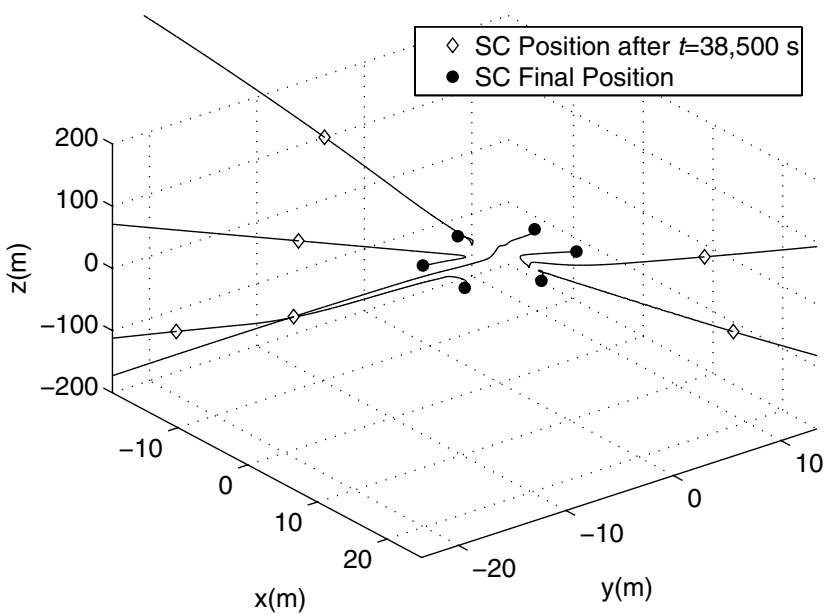

Fig. 6 Simulation of a swarm of satellites reaching an hexagonal regular formation. Second approaching phase. 


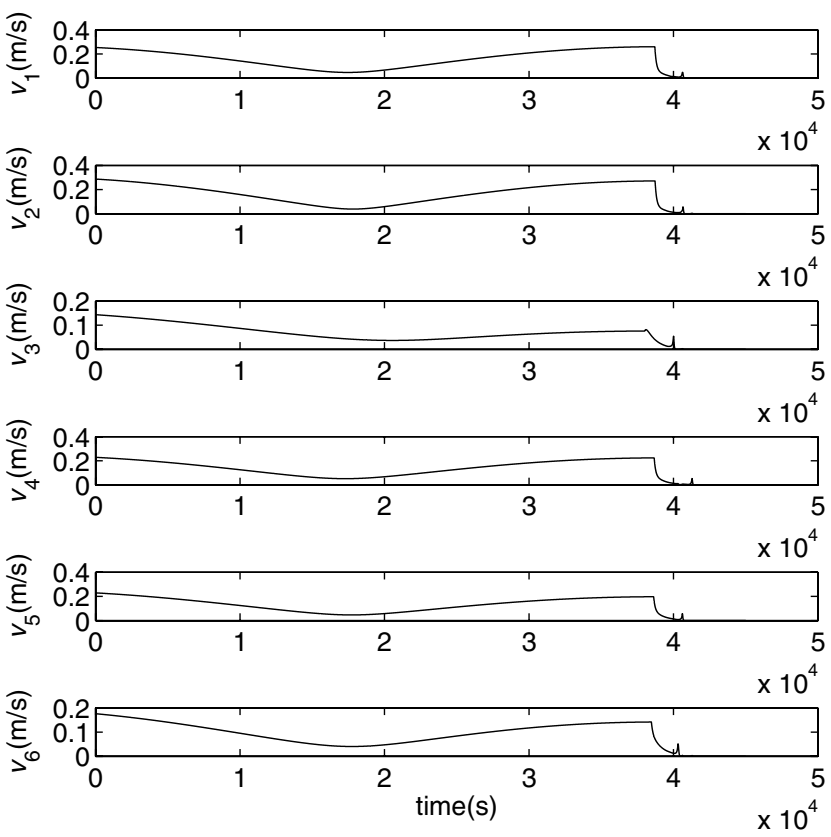

Fig. 7 Velocities of the satellites along the trajectories.

magnitudes of the spacecraft velocities are shown in Fig. 7. The different phases described at the beginning of this section are visible in this chart. Starting from a condition of zero initial velocity, the spacecraft first tracked the ballistic trajectories that brought them into the vicinity of the target configuration. In the last phase of the maneuver (roughly after $38 \times 10^{5}$ s) the satellites reached the edge of the inner part of the kinematical field. In this last phase of the acquisition maneuver, the spacecraft first decelerated and then accelerated again to acquire the final position. The expensive phases of the whole procedure in terms of propellant consumption were the very first seconds, when the engines were constantly saturated to reach a ballistic trajectory (Fig. 8), and the last part of the formation acquisition, when the gravity gradient was no longer considered in the definition of the desired velocities (Fig. 9). It was in this phase that each agent chose its final position and navigated toward it. An average $\Delta v$ of about $0.55 \mathrm{~m} / \mathrm{s}$ was required in this particular maneuver by each agent. During the simulation the minimum occurring intervehicle distance was $5.48 \mathrm{~m}$.
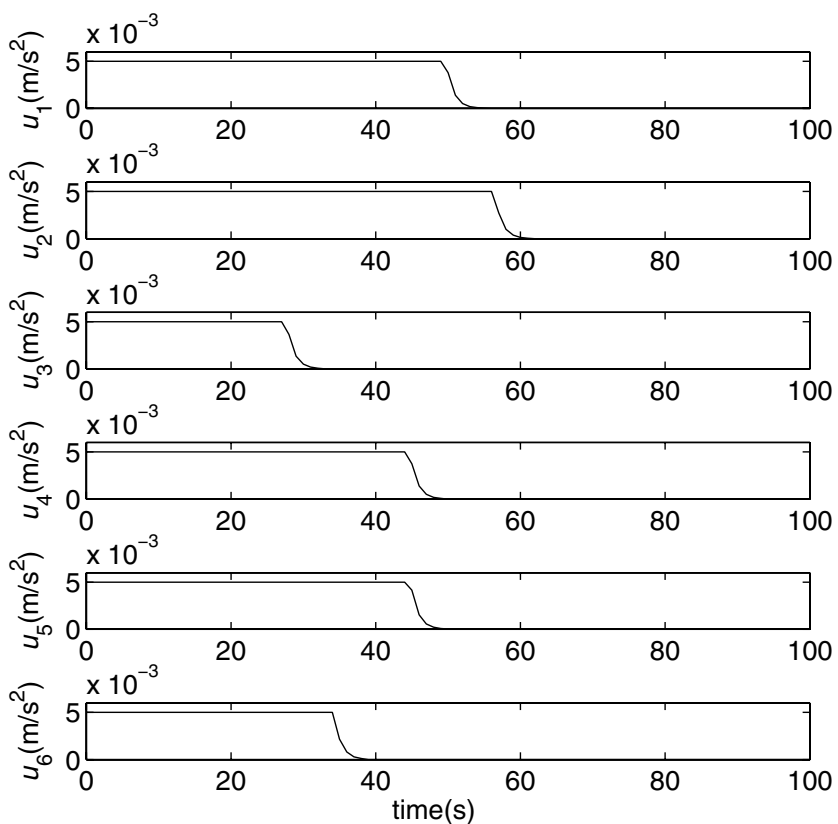

Fig. 8 Control profiles during the first $100 \mathrm{~s}$.
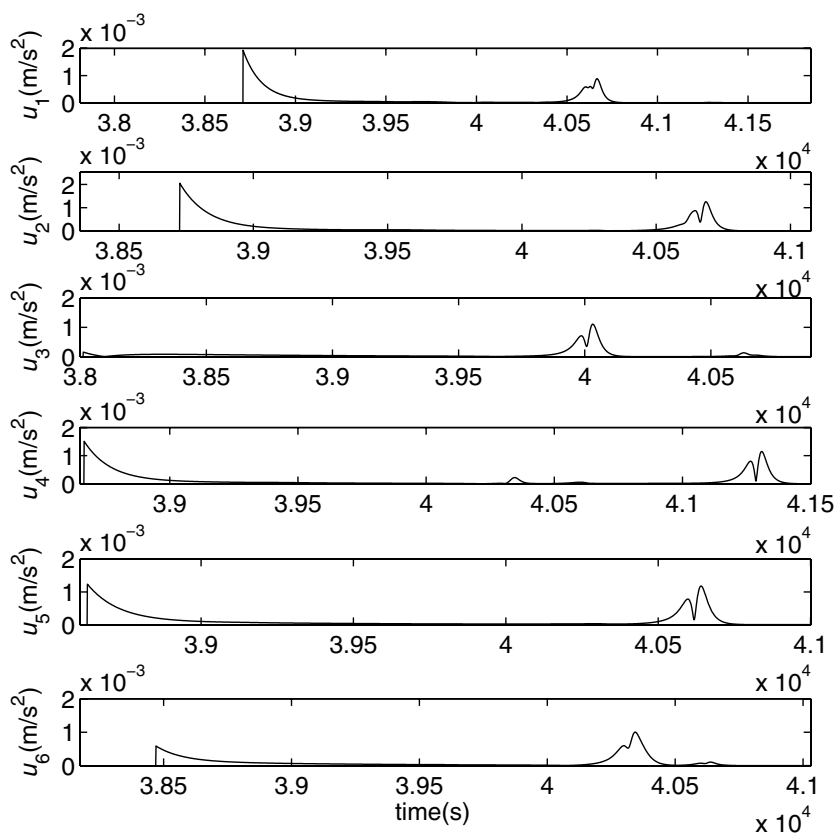

Fig. 9 Control profiles during the last $3500 \mathrm{~s}$.

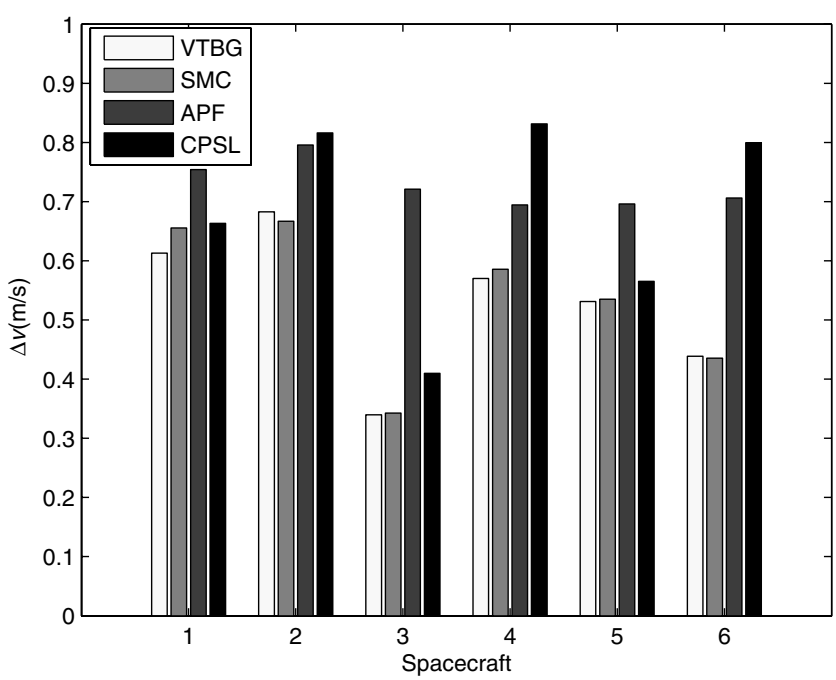

Fig. 10 Propulsion requirements for the different thrusting strategies proposed.

In Fig. 10 the different feedbacks introduced are considered and compared in terms of propellant consumption for this particular simulation. This simulation shows how the SMC and the VTBG feedback are outperforming the CPSL and the APF feedbacks. This result was confirmed also by many other numerical simulations.

\section{Towards a Complex Lattice}

To plan and control its path according to the equilibrium shaping approach, each satellite is required to detect the position of the neighboring spacecraft. This sensing capability may (in principle) be provided in different ways, according to the particular mission considered. Exploration missions [3] would probably use differing solutions for low-Earth-orbit (LEO) formation flying missions or GEO self-assembly concepts [2]. Large swarms of spacecraft are sometimes also considered for scientific missions in which multiple space platforms act in consort, collecting data to achieve a single science objective [25]. We show here how a careful use of intersatellite communications may overcome the limitations in the possible final geometries achievable by the equilibrium shaping approach. We consider a swarm of satellites divided into seed and 
nonseed agents. Each seed is preassigned to a number of nonseed agents. The nonseed agents need to know to which seed they have been preassigned and the shape of the configuration they have to acquire. On the other hand the seeds need only know the shape of their desired configuration. We introduce a new avoid behavior named "inner avoid":

$$
\boldsymbol{v}_{i}^{\text {Avoid }_{\text {in }}}=\sum_{j} b_{\text {in }} \psi_{A}\left(\left\|\boldsymbol{x}_{i}-\boldsymbol{x}_{j}\right\|, k_{A_{\text {in }}}\right)\left(\boldsymbol{x}_{i}-\boldsymbol{x}_{j}\right)
$$

where $k_{A_{\text {in }}}<k_{A}$. Each spacecraft belonging to the swarm can evaluate its own desired velocity field according to the expression

$$
\boldsymbol{v}_{d_{i}}=\boldsymbol{v}_{i}^{\text {Avoid }}+\boldsymbol{v}_{i}^{\text {Avoid }_{\mathrm{in}}}+\boldsymbol{v}_{i}^{\text {Dock }}+\boldsymbol{v}_{i}^{\text {Gather }}
$$

where $\boldsymbol{v}_{i}^{\text {Avoid }}$ acts only between spacecraft belonging to the same group. The seeds plan their path ignoring the nonseed agents (except for the inner collision avoidance). They evaluate and track their desired velocities $\boldsymbol{v}_{s_{i}}$ and communicate it to the nonseed agents belonging to their group. The nonseed agents evaluate their desired velocities $\boldsymbol{v}_{a_{j}}$ using the equilibrium shaping approach but in a frame attached to the seed so that they will eventually acquire their target formation around the seed. The control system of the nonseed agents will therefore track a velocity given by the sum of the two contributions: $\boldsymbol{v}_{\text {tot }_{j}}=\boldsymbol{v}_{s_{i}}+\boldsymbol{v}_{a_{j}}$. Playing with the possible formation achievable by the equilibrium shaping it is possible to build a scheme that can build a number of complex lattices. The inner avoid behavior ensures that agents belonging to different groups will not collide. We define $\underline{d}$ as the minimum distance between two spacecraft in the chosen target configuration. To be able to apply the equilibrium shaping formula in Eq. (8), the inner avoid behavior must be negligible at a length scale $\underline{\bar{d}}$. This can be obtained by finding $k_{A_{\text {in }}}$ so that

$$
\forall i,\left\|\boldsymbol{v}_{i}^{\text {Avoid }_{\text {in }}}\left(\underline{d}, k_{A_{\text {in }}}\right)\right\| \ll \min \left(\left\|\boldsymbol{v}_{i}^{\text {Avoid }}\right\|,\left\|\boldsymbol{v}_{i}^{\text {Dock }}\right\|,\left\|\boldsymbol{v}_{i}^{\text {Gather }}\right\|\right)
$$

When the final configuration is acquired, $\boldsymbol{v}_{i}^{\text {Avoid }_{\text {in }}}$ is the only component in Eq. (27) that defines an interaction between nonseed agents belonging to different groups. Thus the condition in Eq. (28) ensures that the equilibrium shaping approach is still valid and that no conflicts arise in the swarm among different groups of agents.

In the following paragraphs the principal features of this pathplanning scheme are shown through two different examples. To simplify the simulations, each agent is assumed to have perfect control, that is, it can perfectly track the desired kinematical field. The desired kinematical field does not take into account the gravity gradient. We take as a first example a group of 72 agents divided into eight groups of eight nonseed agents, and a group of eight agents considered as seeds. The agents are randomly placed in the proximity
Table 2 Parameters for the simulation with 72 spacecraft acquiring a lattice formation

\begin{tabular}{lcc}
\hline & Seeds & Nonseeds \\
\hline$b, \mathrm{~s}^{-1}$ & 0.02751 & 0.0215 \\
$d, \mathrm{~s}^{-1}$ & 0.0075 & 0.0059 \\
$c, \mathrm{~s}^{-1}$ & $9.4964 \times 10^{-5}$ & $7.4190 \times 10^{-5}$ \\
$k_{A}, \mathrm{~m}$ & 40 & 8 \\
$k_{D}, \mathrm{~m}$ & 40 & 8 \\
\hline \hline
\end{tabular}

of a large target configuration with the shape of a cubic lattice. The average distance of the agents from the center of the desired formation at $t=0$ is $477.7 \mathrm{~m}$ and the standard deviation is $137.8 \mathrm{~m}$. In the final configuration the target positions form a cube of side $L=80 \mathrm{~m}$, whereas each group of nonseed spacecraft forms a cube of side $l=16 \mathrm{~m}$ around each agent. The minimum distance between two agents belonging to the formation is $\underline{d}=l$, and letting $k_{A_{\text {in }}}=4 \mathrm{~m}$, the condition in Eq. (28) is satisfied. For the cube formation the equilibrium shaping formula reduces to a single uncoupled equation in three unknowns. It is then possible to choose independently the values of the parameters $b$ and $d$ to select the formation acquisition time. The parameters chosen for this simulation are listed in Table 2 . The final formation is acquired in approximately $10,000 \mathrm{~s}$. Figure $11 \mathrm{a}$ shows the actual assembly sequence as returned by one of the performed simulations.

In the second example we drive the assembly of a large structure in space. The objective is to form a flat structure that can be used as a large reflector for deep space observation or solar power collection purposes [2]. The reflector assembly can be accomplished by first applying the equilibrium shaping technique to acquire a given target formation and then exploiting a docking algorithm to assemble the structure. Let us consider a swarm of 49 spacecraft, with a group of seven homogeneous seeds and seven groups of six nonseed agents. The target configuration is a lattice formation in which the seeds form a centered hexagon of side $R=60 \mathrm{~m}$ while the nonseed agents are disposed around each seed forming a hexagon of side $r=16 \mathrm{~m}$. In the target configuration the minimum distance between two agents belonging to different groups is $r$. A value of the $\boldsymbol{v}^{\text {Avoid }_{\text {in }}}$ characteristic length of $k_{A_{\text {in }}}=4 \mathrm{~m}$ satisfies the condition in Eq. (28). All the seed target positions located at the vertices of the hexagon (named $\boldsymbol{\xi}_{1}, \ldots, \boldsymbol{\xi}_{6}$ ) are equivalent with respect to a rotation of $60 \mathrm{deg}$ around the hexagon center and belong to a formation symmetry axis. The remaining target position (named $\xi_{7}$ ) is located at a formation symmetry point. Therefore, by setting $d_{1}=\ldots=d_{6}=d$ and $c_{1}=\ldots=c_{6}=c$, the equilibrium shaping formula is recast into a single scalar equation in the four unknowns $c, d, c_{7}, d_{7}$. Recall that the $b$ parameter is not included in the set of independent variables of the equilibrium shaping formula but rather is considered as an

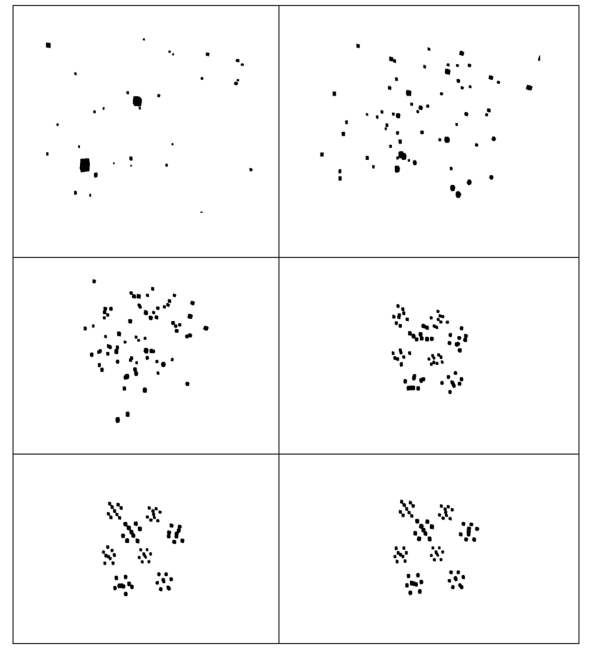

a)

Fig. 11 Assembly sequence for two lattices.

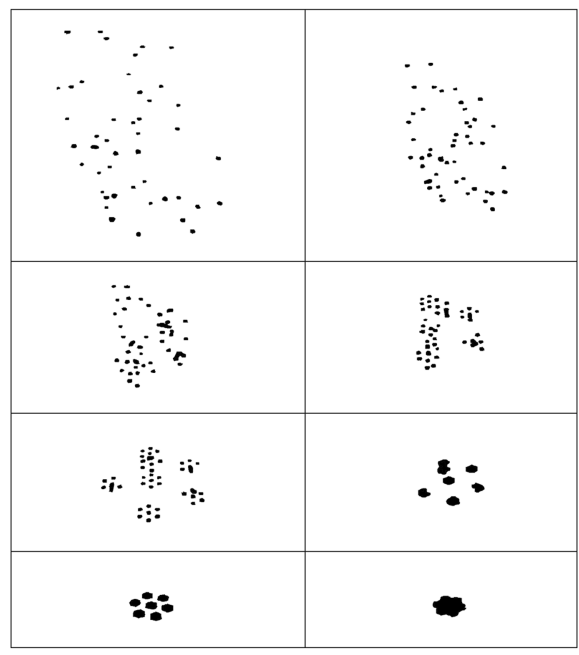

b) 
Table 3 Parameters for the simulation with 49 spacecraft assembling a flat structure in space

\begin{tabular}{lcc}
\hline \hline & Seeds & Nonseeds \\
\hline$b, \mathrm{~s}^{-1}$ & 0.0458 & 0.0430 \\
$d, \mathrm{~s}^{-1}$ & 0.0333 & 0.0156 \\
$d_{7}, \mathrm{~s}^{-1}$ & 0.0125 & - \\
$c, \mathrm{~s}^{-1}$ & $0.8712 \times 10^{-4}$ & $0.8355 \times 10^{-4}$ \\
$c_{7}, \mathrm{~s}^{-1}$ & $0.2345 \times 10^{-4}$ & - \\
$k_{A}, \mathrm{~m}$ & 30 & 8 \\
$k_{D}, \mathrm{~m}$ & 30 & 8 \\
\hline \hline
\end{tabular}

independent parameter to be chosen by the system designer. The values of $d, c, d_{7}, c_{7}$, and $b$ used in this simulation for all the agents are listed in Table 3 . They have been chosen heuristically to reduce the occurrence of local minima and to select a certain acquisition time. Figure $11 \mathrm{~b}$ visualizes the different phases of the assembly procedure. At the initial time (Fig. 11b, frame 1) the 49 agents are distributed in a cloud with an average distance with respect to the swarm center of mass of $453.8 \mathrm{~m}$ and with a standard deviation of $147.1 \mathrm{~m}$. The swarm acquires the desired lattice formation in approximately $20,000 \mathrm{~s}$ following the path planned according to the equilibrium shaping technique (Fig. 11b, from frame 2 to frame 7). Once the final formation is acquired the spacecraft can perform the docking maneuver, relying upon an autonomous docking procedure (Fig. 11b, frame 8). A detailed discussion of the docking phase is out of the scope of the present paper.

As a final remark we point out that the position of each agent in the final formation is not completely preassigned, and the target selection problem, as defined in Sec. III, is autonomously solved in real time by the agents during the acquisition maneuver and taking into account the seed, nonseed associations. All in all, the swarm composed of $N$ seeds and $N$ groups of $n$ agents autonomously decides which configuration to acquire in the space among the $(N !)(n !)^{N}$ possibilities. The final configuration is thus chosen from $2.8163 \times$ $10^{41}$ possible configurations in the simulation shown in Fig. 11a and from $5.0554 \times 10^{23}$ possibilities in the simulation shown in Fig. $11 \mathrm{~b}$. The problem of self-assembling a hexagonal lattice has recently been solved also at a molecular level by using an inverse method by Rechtsman et al. [26]. Their technique is, however, not applicable to satellites navigation and path planning, as it is inherently twodimensional. The similarity with the equilibrium shaping is anyway important, and proves that inverse methods for self-assembly are interesting in connection with different fields.

\section{Conclusions}

The equilibrium shaping technique introduced in this paper represents a novel approach for satellite collaborative path planning. It constitutes a rather simple algorithm that makes use only of local sensory information and limited computational resources. For a number of final configurations described in this paper the equilibrium shaping technique allows us not only to safely acquire and maintain the formation, but also to autonomously assign a target position to each satellite. Because the method is based on a behavioral-based approach defined in terms of desired velocities, a velocity tracking algorithm has to be implemented. Standard methods such as slidingmode control, Q-guidance, artificial potential, and cross-product steering law are all suitable for this purpose. Although the method is not limited to any particular environment, simulations of these different feedbacks showed that the performances in terms of propellant for applications in the geostationary orbit are well within our technological capabilities. Complicated lattice geometries may also be acquired using the equilibrium shaping technique at the cost of adding some extra intersatellite communication capability.

\section{Acknowledgment}

The authors wish to acknowledge the contributions made by Mark Ayre in developing the ideas included in this paper.

\section{References}

[1] Kube, C., and Zhang, H., "Collective Robotic Intelligence: From Social Insects to Robots," Adaptive Behavior, Vol. 2, No. 2, 1993, pp. 189218.

[2] Ayre, M., Izzo, D., and Pettazzi, L., "Self Assembly in Space Using Behaviour Based Intelligent Components," TAROS, Towards Autonomous Robotic Systems, Imperial College, London, Sept. 2005.

[3] D'Arrigo, P., and Santandrea, S., "The APIES Mission," ASTRIUM Ltd./ESA-ESTEC Feasibility Study A0/1-3846/02/NL/JA, Stevenage, U.K., 2004.

[4] Curtis, S., Mica, J., Nuth, J., Marr, G., Rilee, M., and Bath, M., "ANTS (Autonomous Nano-Technology Swarm): An Artificial Intelligence Approach to Asteroid Belt Resource Exploration," IAF Paper 00Q.05.08, Oct. 2000.

[5] Khatib, O., "Real-Time Obstacle Avoidance for Manipulators and Mobile Robots," The International Journal of Robotics Research, Vol. 5, No. 1, 1986, pp. 90-98.

[6] McInnes, C., "Autonomous Rendezvous Using Artificial Potential Functions," Journal of Guidance, Control, and Dynamics, Vol. 18, No. 2, 1995, pp. 237-241.

[7] McQuade, F., "Autonomous Control for On-Orbit Assembly Using Artificial Potential Functions,” Ph.D. Thesis, Faculty of Engineering, University of Glasgow, Glasgow, Scotland, U.K., 1997.

[8] Sato, K., "Dead-Lock Motion Path Planning Using The Laplace Potential Field," Advanced Robotics, Vol. 17, No. 5, 1993, pp. 449461.

[9] Keymeulen, D., and Decuyper, J., "The Fluid Dynamics Applied to Mobile Robot Motion: The Stream Field Method," Proceedings of the IEEE International Conference on Robotics and Automation, IEEE Publications, Piscataway, NJ, 1994, pp. 378-386.

[10] Chang, H., "A New Technique to Handle Local Minimum for Imperfect Potential Field Based Path Planning," Proceedings of the IEEE International Conference on Robotics and Automation, Vol. 1, IEEE Publications, Piscataway, NJ, 1996, pp. 108-112.

[11] Schoner, G., and Dose, M., "A Dynamics Systems Approach to Task Level Systems Integration used to Plan and Control Autonomous Vehicle Motion," Robotics and Autonomous Systems, Vol. 10, No. 4, 1992, pp. 253-267.

[12] Ren, W., and Beard, R., "A Decentralized Scheme for Spacecraft Formation Flying via the Virtual Structure Approach," Journal of Guidance, Control, and Dynamics, Vol. 27, No. 1, Jan. 2004, pp. 7382.

[13] Lawton, J., Young, B., and Beard, R., "A Decentralized Approach to Elementary Formation Maneuvers," IEEE Transactions on Robotics and Automation, Vol. 17, No. 6, 2003, pp. 933-941.

[14] Campbell, M., "Planning Algorithm for Multiple Satellite Clusters," Journal of Guidance, Control, and Dynamics, Vol. 26, No. 5, 2003, pp. 770-780.

[15] Pirjanian, P., "Behavior Coordination Mechanism State of the Art," University of Southern California, Tech. Report IRIS-99-375, Institute for Robotics and Intelligent Systems, School of Engineering, Los Angeles, Oct. 1999.

[16] Gazi, V., "Swarm Aggregations Using Artificial Potentials and Sliding Mode Control," Proceedings of the IEEE Conference on Decision and Control, IEEE Publications, Piscataway, NJ, Dec. 2003, pp. 28482853.

[17] Gazi, V., and Passino, K., "A Class of Attraction/Repulsion Functions for Stable Swarm Aggregations," International Journal of Control, Vol. 77, No. 18, 2004, pp. 1567-1579.

[18] Large, E., Christensen, H., and Bajcsy, R., "Dynamic Robot Planning: Cooperation Through Competition," Proceedings of the IEEE International Conference on Robotics and Automation, Vol. 3, IEEE Publications, Piscataway, NJ, May 1997, pp. 2306-2312.

[19] Izzo, D., and Pettazzi, L., "Equilibrium Shaping: Distributed Motion Planning for Satellite Swarm," Proceedings of the 8th International Symposium on Artificial Intelligence, Robotics and Automation in Space, SP-603, ESA Publications Division, Noordwijk, The Netherlands, Sept. 2005.

[20] Clohessy, W., and Wiltshire, R., "Terminal Guidance System for Satellite Rendezvous," Journal of the Aerospace Sciences, Vol. 27, No. 9, Sept. 1960, pp. 653-658.

[21] Battin, R. H., An Introduction to the Mathematics and Methods of Astrodynamics, AIAA Educational Series, AIAA, New York, 1987, pp. $23-29$.

[22] De Carlo, R., Zak, S., and Drakunov, S., The Control Handbook, CRC Press/IEEE Press, Boca Raton, FL, 1996, pp. 941-951, Chap. 57.5.

[23] Young, K., Utkin, V., and Ozguner, U., "A Control Engineer's Guide to Sliding Mode Control," IEEE Transactions on Control Systems 
Technology, Vol. 7, No. 3, 1999, pp. 328-342.

[24] Romanelli, C., Natarajan, A., Schaub, H., Parker, G., and King, L., "Coulomb Spacecraft Voltage Study due to Differential Orbital Perturbation," AAS Paper 06-123, Jan. 2006.

[25] Magness, R., "Short-Range RF Wireless (Proximity) Networks Technology Assessment for Space Application," ESA, Technology
Dossier TOSE-1B-DOS-4 TOS-EDD/2004.3/RM, Noordwijk, The Netherlands, 2006.

[26] Rechtsman, M., Stillinger, F., and Torquato, S., "Optimised Interactions for Targeted Self-Assembly: Application to a Honeycomb Lattice,” Physical Review Letters, Vol. 95, No. 228301, Nov. 2005. 